\title{
MERS PUI Surveillance and Restrospective Identification in ESSENCE-FL, 2013-2015
}

\author{
Julia G. Munroe*, Rachael Straver, Heather Rubino, Scott Pritchard, David Atrubin and \\ Janet J. Hamilton
}

Florida Department of Health, Tallahassee, FL, USA

\section{Objective}

To retrospectively identify initial emergency department (ED) and urgent care center (UCC) visits for Florida's Middle East respiratory syndrome coronavirus disease (MERS-CoV) patients under investigation (PUIs) in the Florida Department of Health's (DOH) syndromic surveillance system, the Electronic Surveillance System for the Early Notification of Community-based Epidemics (ESSENCE-FL), using information gathered from PUI case report forms and corresponding medical records for the purpose of improving syndromic surveillance for MERS-CoV. The results of this study may be further utilized in an effort to evaluate the current MERS-CoV surveillance query.

\section{Introduction}

Human MERS-CoV was first reported in September 2012. Globally, all reported cases have been linked through travel to or residence in the Arabian Peninsula with the exception of cases associated with an outbreak involving multiple health care facilities in the Republic of Korea ending in July 2015. While the majority of MERS-CoV cases have been reported in the Arabian Peninsula, several cases have been reported outside of the region. Most cases are believed to have been acquired in the Middle East and then exported elsewhere, with no or rare instances of secondary transmission. Two cases of MERS-CoV were exported to the United States and identified in May 2014. One of these cases traveled from Saudi Arabia to Florida.

DOH conducts regular surveillance for MERS-CoV through the investigation of persons with known risk factors. PUIs have most often been identified by physicians reporting directly to local health departments and by DOH staff regularly querying ED and UCC chief complaint data in ESSENCE-FL. ESSENCE-FL currently captures data from 265 EDs and UCCs statewide and has been useful in identifying cases associated with reportable disease and emerging pathogens.

\section{Methods}

From 2013-2015 DOH identified and investigated 62 suspected cases of MERS-CoV, including one confirmed case in May 2014. Specimens were collected from all 62 patients under investigation (PUIs) and 61 were ruled out. Of the 61 PUIs who were ruled out, ten were part of the contact investigation initiated following the identification of MERS-CoV in May 2014 and were not included in this analysis. DOH utilizes a MERS-CoV PUI case report form to collect data regarding demographics, clinical presentation, and risk factors. Retrospectively, additional documents including medical records and discharge summaries were gathered and utilized to evaluate PUIs identified in ESSENCE-FL.

Name of the facility where PUIs presented, date and time of visit, age at event, and sex were identified using PUI case report forms and corresponding medical records and discharge summaries. Visit details for each of the identified facilities were queried in ESSENCE-FL and pulled for all visits with corresponding age at event and sex for the patient's visit date. Additional PUI information including chief complaint, discharge diagnosis, ZIP code, race, and ethnicity were gathered for the purpose of matching corresponding ESSENCE-FL data fields. ESSENCE-FL visit details were narrowed by ZIP code (or lack of ZIP code for residents of other countries) and match details were recorded and evaluated. The fields examined were not always complete in ESSENCE-FL. Visits were considered matches when all available data in the fields examined were consistent with information obtained in the PUI case report form and available medical records and discharge summaries.

\section{Results}

Of the 52 PUIs included in this analysis, 39 sought treatment at facilities participating in ESSENCE-FL at their time of visit. Comparing information obtained from PUI documents with data provided in ESSENCE-FL, 30 ED visits were successfully matched to PUIs, including an initial ED visit for the patient with a confirmed case of MERS-CoV.

\section{Conclusions}

Following preliminary identification, all matches are to be confirmed with the appropriate hospitals. Future work to examine the chief complaints associated with patients' initial ED visits identified in ESSENCE-FL will serve as a way to validate and improve upon the query currently being used as a surveillance tool for MERS-CoV. Detailing these methods also has value in the replication of this study for other diseases and in the development and validation of other disease-specific queries. Summarizing the reasons why PUIs were unable to be matched to ESSENCE-FL visits is also useful in improving system robustness.

\section{Keywords}

Surveillance; Syndromic; MERS; Investigation; ESSENCE

\section{*Julia G. Munroe}

E-mail: julia.munroe@flhealth.gov 